\title{
THE EFFECTS OF POLYGLYCEROL ESTERS ON PALM OLEIN FRACTIONATION
}

\author{
YOONG JUN HAO*; SAW MEI HUEY*; YEOH CHEE BENG*; SIVARUBY KANAGARATNAM*; LUQMAN \\ CHUAH ABDULLAH $^{\star \star}$ and THOMAS CHOONG SEAN YAW**
}

\begin{abstract}
This study was undertaken to investigate the effects of adding $0.1 \%(w / w)-0.9 \%(w / w)$ polyglycerol esters (PGE) on palm olein fractionation based on crystal nucleation, crystal growth and product quality aspects. Palm olein (IV 56) with PGE additives was subjected to crystallisation at a temperature of $13^{\circ} \mathrm{C}$ for 335 min. The crystal nucleation and its growth were monitored using focused beam reflectance measurement (FBRM) technique, during which the in-line changes of crystal size distribution during the process were analysed. The fractionated products were analysed for their iodine value (IV) and triacylglycerol (TAG) composition. The FBRM results showed that the PGE promoted crystal nucleation coupled with reduced formation of undesired large crystals during the crystallisation stage. The product analysis also showed that the PGE discouraged the formation of olein entrainment in the solid fraction but promoted the formation of 1, 3-dipalmitoyl-2-oleoyl-glycerol (POP) content in the solid fraction. In addition, PGE also slowed the formation of 1-palmitoyl-2,3-dioleoy-glycerol (POO). The optimal dosage that offered best performance was $0.3 \%$ (w/w) PGE which gave the most medium sized crystals and best palm mid fraction product with lowest IV and highest content of POP. These results implied that PGE is a nucleation enhancing and crystal growth retarding additive in palm olein crystallisation at $0.3 \%$ (w/w) dosage.
\end{abstract}

Keywords: palm olein, fractionation, polyglycerol esters, crystallisation, palm mid fraction.

Date received: 27 June 2018; Sent for revision: 5 July 2018; Received in final form: 2 November 2018; Accepted: 10 April 2019.

\section{INTRODUCTION}

Oil palm produces two types of oils, namely crude palm oil from mesocarp and crude palm kernel oil from the kernel. Palm oil consists mainly of glycerides and small portions of phytonutrients. In order to render the oils for different applications, these glycerides need to be modified into palm olein [liquid fraction of palm oil which contains more low melting triacylglycerols (TAG)] and palm stearin

\footnotetext{
* Malaysian Palm Oil Board,

6 Persiaran Institusi, Bandar Baru Bangi,

43000 Kajang, Selangor, Malaysia.

E-mail: jhyoong@mpob.gov.my

** Faculty of Chemical and Engineering,

Universiti Putra Malaysia, 43400 UPM Serdang,

Selangor, Malaysia.
}

(solid fraction of palm oil which contains more high melting TAG) with different iodine values (IV). Fractionation is a process that separates high melting TAG from low melting TAG of oils and fats (Metin and Hartel, 2005). This is a natural process as compared to other fat modification processes such as interesterification and hydrogenation. Removing certain amounts of higher melting components from oils and fats will cause the remaining oil to be more liquid such that it can be used at lower temperatures as a liquid oil and it can also be blended with other liquid oil to enhance further its cold stability characteristic (Deffense, 1985). Dry fractionation involves two major parts, i.e. crystallisation and filtration.

The dry fractionation is however less efficient than solvent or detergent fractionation processes 
(Timms, 2005) especially in fractionating the palm olein, mainly due to slow crystallisation behaviour of palm olein and olein entrainment in the solid fraction. Difficulty in obtaining a slurry with suitable crystal size during crystallisation makes this process very challenging. Generally, bigger crystals are desired in the fractionation as they are easier to be filtered in membrane filters. However, this tends to make the crystals agglomerate or group together in clumps occluding part of the liquid and, as a result, some olein will be lost in the stearin fraction (Suria, 1996). This olein loss phenomenon is popularly referred to as olein entrainment. The olein entrainment becomes less when crystal size reduces. In this context, many researches have been carried out to improve the fractionation efficiency by reducing production time and enhancing the crystallisation techniques to avoid olein entrainment especially for multiple stages of fractionation.

Polyglycerol esters (PGE) was introduced in this study as the additive agent for enhancing the palm olein fractionation process. Previous study showed that by addition of PGE promoted formation of more uniform crystals, which helped to increase yield of the palm olein products from the palm oil fractionation process at $24^{\circ} \mathrm{C}$ (Kuriyama et al., 2011) and significantly increased the crystallisation temperature of PMF (Sakamoto et al., 2004). Studies by Sakamoto et al. (2004) and Zhang et al. (2016) suggested that saturated fatty acids in the PGE (namely palmitic and stearic acids) significantly increased crystallisation temperature. A recent study on the effect of PGE on palm oil crystallisation by Saw et al. (2017) using focused beam reflectance measurement (FBRM) and differential scanning calorimetry (DSC) showed that PGE additive increased the total crystal population as compared with the control and the effects were even significant when PGE was increased from $0.5 \%$ to $0.7 \%$. Saw et al. (2017) also observed that the dosages of PGE prevented the formation of extra large crystals with sizes ranging from $293-1000 \mu \mathrm{m}$ and lowered the number of large crystals $(100-251 \mu \mathrm{m})$. This characteristic of inhibiting crystal growth to a larger size favours the fractionation process and reduces the possibility of olein entrainment. In addition, $0.7 \%$ PGE was observed to be the most effective in nucleation promotion as compared with other dosages.

In general, previous studies in PGE showed positive effects in fractionation enhancement where it promoted nucleation which resulted in the increase of crystal population and inhibited the growth of large crystals which is unfavourable in the filtration process. The PGE molecule behaved as a foreign surface to promote the formation of more nuclei in the crystallisation (Sato et al., 1999). In the light of the possible findings in previous studies, it is hypothesised that PGE additive can be one of the possible options to enhance the crystallisation which will reduce the fractionation time and promote formation of even-sized palm olein crystals. The effects of PGE additive on the crystallisation of palm olein require studies to determine its optimum dosage and crystallisation mechanism. Currently, studies on PGE are limited and there is no available data on the effects of PGE additive on palm olein crystallisation (Saw et al., 2017; Sakamoto et al., 2005).

\section{MATERIALS AND METHODS}

\section{Materials}

Commercial-grade refined, bleached and deodourised palm olein (RBDPOo) was purchased from Lam Soon Edible Oil Sdn Bhd (Johor, Malaysia). The PGE additive with a mixture of fatty acids of palmitic acid, stearic acid and oleic acid was provided by Sakamoto Yakuhin Kogyo Co. Ltd (Osaka, Japan). The melting point of the PGE was $42.8^{\circ} \mathrm{C}$ (Kuriyama et al., 2011). PGE is a crystallisation aid which has been introduced to promote nucleation, provides a surface area to additional crystal growth and to make the overall lipid molecule more readily come together into mixed crystals. The hydrophiliclipophilic balance (HLB) value of the PGE is 1.6 as calculated in accordance with Griffin's equation (Kuriyama et al., 2011). PGE was added to $750 \mathrm{~g}$ RBDPOo at the concentrations of $0.1 \%(\mathrm{w} / \mathrm{w}), 0.3 \%$ $(\mathrm{w} / \mathrm{w}), 0.5 \%(\mathrm{w} / \mathrm{w}), 0.7 \%(\mathrm{w} / \mathrm{w})$ and $0.9 \%(\mathrm{w} / \mathrm{w})$.

\section{Crystallisation}

The pre-melted $750 \mathrm{~g}$ of RBDPOo and the additive was loaded into 1 L-stirred, jacketed glass Mettler Toledo Labmax reactor with FBRM that was equipped with a glass anchor agitator, stirred at a rate of $30 \mathrm{rpm}$. During the crystallisation, RBDPOo was cooled to the desired temperature of $13^{\circ} \mathrm{C}$ for a period of $335 \mathrm{~min}$. Crystal nucleation and its changes were observed from $335 \mathrm{~min}$ until $470 \mathrm{~min}$ (total $135 \mathrm{~min}$ ) when the crystallisation was held under isothermal conditions for a period of $135 \mathrm{~min}$ after which the RBDPOo slurry was subjected to a filtration process to separate the liquid and solid fractions.

\section{Crystal Size Distribution by FBRM}

During the crystallisation process, the oil structure was observed in $1 \mathrm{~L}$-stirred, jacketed glass Mettler Toledo Labmax reactor equipped with a Lasentec D600L probe (manufactured by Mettler Toledo, Schwerzenbach, Switzerland in 2006). The probe was used to monitor the particle counts and size distribution within the crystalliser. 
Studies on nucleation and crystal growth with or without PGE were conducted during the process. Data were collected using 90 logarithmicallyspaced channels over the size range of 1-1000 $\mu \mathrm{m}$. The size distribution of crystals, expressed as chord length by FBRM, was recorded at every $30 \mathrm{~s}$ during the run. The crystal nucleation was determined by the observation on particle counts and mean particle sizes using the FBRM. The information of total particle counts can be directly related to nucleation (Yu et al., 2008). Crystal counts were categorised into five classes: 1-5 $\mu \mathrm{m}$ (extra small crystal, XSc), 10-23 um (small crystal, Sc), 29-86 um (medium crystal, Mc), 100-251 $\mu \mathrm{m}$ (large crystal, Lc) and 293-1000 $\mu \mathrm{m}$ (extra large crystal, XLc). Each experiment was done in duplicate. The same procedure was conducted for RBDPOo crystallisation at all additive dosages including the control run without PGE.

\section{Analyses of Samples}

TAG composition of the samples was determined by using reversed-phase high performance liquid chromatography (HPLC) (Agilent Technologies, Wilmington, DE, USA). The instrument was equipped with a refractive index detector and Lichrospher ${ }^{\circledR 1} 100$ RP-18 column ( $250 \mathrm{~mm} \times 4.6 \mathrm{~mm})$ with $5 \mu \mathrm{m}$ particle size. Oil samples, $0.045 \mathrm{~g}$, were weighed and diluted in acetone for injection into the HPLC column. The mobile phase used in the analysis was a mixture of 3:1 ratio of acetone and acetonitrile at a flow rate of 1 $\mathrm{ml} \mathrm{min}{ }^{-1}$. The TAG peaks identification was done by referring to Ghazali et al. (1995) and Swe et al. (1995). The most abundant TAG in RBDPOo are 1-palmitoyl2,3-dioleoyl-glycerol (POO) and 1-3-dipalmitoyloleoyl-glycerol (POP).

IV of the samples and fractionation products, i.e. double fractionated palm olein (DFPOo) and palm mid fraction (PMF) were analysed according to MPOB Test Method p3.2 (MPOB, 2005).

\section{Statistical Analysis}

The FBRM data were analysed using the analysis of variance (one-way ANOVA) to determine the significance of the additive dosages on overall crystal growth rate, total particle count and mean particle size at $95 \%(\mathrm{w} / \mathrm{w})$ confidence level. The readings from the FBRM on the particle counts and mean particle sizes for $0.1 \%(\mathrm{w} / \mathrm{w}), 0.3 \%(\mathrm{w} / \mathrm{w})$, $0.5 \%(\mathrm{w} / \mathrm{w})$ and $0.7 \%(\mathrm{w} / \mathrm{w})$ PGE additives were analysed using Dunnett method, with $0 \%(\mathrm{w} / \mathrm{w})$ as the control. The particle counts and mean particle sizes were compared with the control to determine whether the differences are statistically significant. The Dunnett's Test was carried out by conducting a Student's t-test between each experimental group and the control group using the Minitab 16.2 software.

\section{RESULTS AND DISCUSSIONS}

\section{Effects of PGE on Crystal Nucleation}

Two important stages in the crystallisation are nucleation and crystal growth. The crystal nucleation is one of the important factors to determine the effectiveness of an additive in enhancing crystallisation. This section discusses the changes of crystal nucleation of RBDPOo during the isothermal holding time from $335 \mathrm{~min}$ to $470 \mathrm{~min}$. The duration of isothermal holding time was $135 \mathrm{~min}$.

Figure 1 shows the effects of PGE on the crystal nucleation at $470 \mathrm{~min}$ and $13^{\circ} \mathrm{C}$, the final step of isothermal crystallisation before filtration. Particle counts and mean crystal size increased when dosage of PGE was increased from control to $0.3 \%$ $(\mathrm{w} / \mathrm{w})$. In general, all PGE dosages increased the total particle counts as compared with the control. Data analysis using Dunnett method indicated that the total counts for all dosages were significantly different compared to the control $(\mathrm{p}=0)$. The particle counts and mean crystal size reached the peak at $0.3 \%(\mathrm{w} / \mathrm{w})$ and slightly decreased from dosage of $0.5 \%(\mathrm{w} / \mathrm{w})$ to $0.9 \%(\mathrm{w} / \mathrm{w})$. This indicates that the dosage of PGE promoted nucleation, and at $0.3 \%$ $(\mathrm{w} / \mathrm{w})$ had the most significant effect on nucleation. PGE induced catalytic crystallisation could result in the formation of a primary heterogeneous nucleation in RBDPOo during the crystallisation. This can be observed from the particle counts (crystal population) which increased drastically when PGE was added as compared with control. These findings agree with the study conducted by Saw et al. (2017), where the rate of particle formation during the oil crystallisation increased when the dosages of PGE were increased. The present study on RBDPOo crystallisation however was found to be more complicated when compared with the palm oil crystallisation study by Saw et al. (2017) due to the complexity of the TAG compositions in RBPOo which had similar melting points. However, at dosages of PGE from $0.5 \%(\mathrm{w} / \mathrm{w})$ to $0.9 \%(\mathrm{w} / \mathrm{w})$ the particle counts declined when compared to $0.3 \%$ $(\mathrm{w} / \mathrm{w})$ as excessive dosage of PGE could inhibit crystal growth. This was partly contributed by the oleic acid present in the PGE where the inhibitory effects of the unsaturated fatty acid on crystallisation was shown to be more profound when the dosages increased. These findings agreed with the previous study where excessive dosages of unsaturated fatty acid or linoleic acid PGE at 10\% (w/w) were found having inhibitory effects on crystallisation (Zhang et al., 2016). Since PGE additive is a crystallisation enhancer, it induces catalytic effect to trigger nucleation in the crystallisation process. Precise and optimum dosing of PGE will be more effective to enhance the crystallisation process as compared to excessive dosages. 
Figure 2 demonstrates the number of particles in different crystal sizes during the crystallisation with or without PGE dosage. Crystal counts were categorized into five classes: 1-5 $\mu \mathrm{m}$ (extra small crystal, XSc), 10-23 $\mu \mathrm{m}$ (small crystal, Sc), 29-86 $\mu \mathrm{m}$ (medium crystal, Mc), 100-251 $\mu \mathrm{m}$ (large crystal, Lc) and 293-1000 $\mu \mathrm{m}$ (extra large crystal, XLc). The findings indicate that PGE mainly promotes the growth of crystal sizes of small and medium sizes. This effect can be seen clearly in the dosage of $0.3 \%$ $(\mathrm{w} / \mathrm{w}), 0.5 \%(\mathrm{w} / \mathrm{w}), 0.7 \%(\mathrm{w} / \mathrm{w})$ and $0.9 \%(\mathrm{w} / \mathrm{w})$. During the final steps before filtration at $470 \mathrm{~min}$ or $135 \mathrm{~min}$ of isothermal holding time, minimum crystals were found in the control (less than 200 counts) and the crystals were mostly medium sizes but their sizes increased when the PGE dosage was increased. The particle counts for medium size crystals of $0.3 \%(\mathrm{w} / \mathrm{w})$ were the highest at 5000 and the increase in particle counts was significant as compared with the control and $0.1 \%(\mathrm{w} / \mathrm{w})$. In addition, $0.3 \%(\mathrm{w} / \mathrm{w})$ had higher number of small crystals as compared with other PGE dosages. This observation agrees with Saw et al. (2017) which showed that lower dosage of PGE has lower rates of particle growth possibly due to minor retardation of nucleation. Based on Normah et al. (2013), formation of crystals with size ranging from 4.34 to $29.29 \mu \mathrm{m}$ which are stable and uniform are important to facilitate separation of the oil from crystals during filtration. This will produce higher quality stearin with minimum oil entrainment (Normah et al., 2013). In the present study, the increased amounts of medium and small crystals facilitated the enhanced quality of the products after filtration.

Crystal nucleation during the isothermal crystallisation (from $335 \mathrm{~min}$ to $470 \mathrm{~min}$ ) was

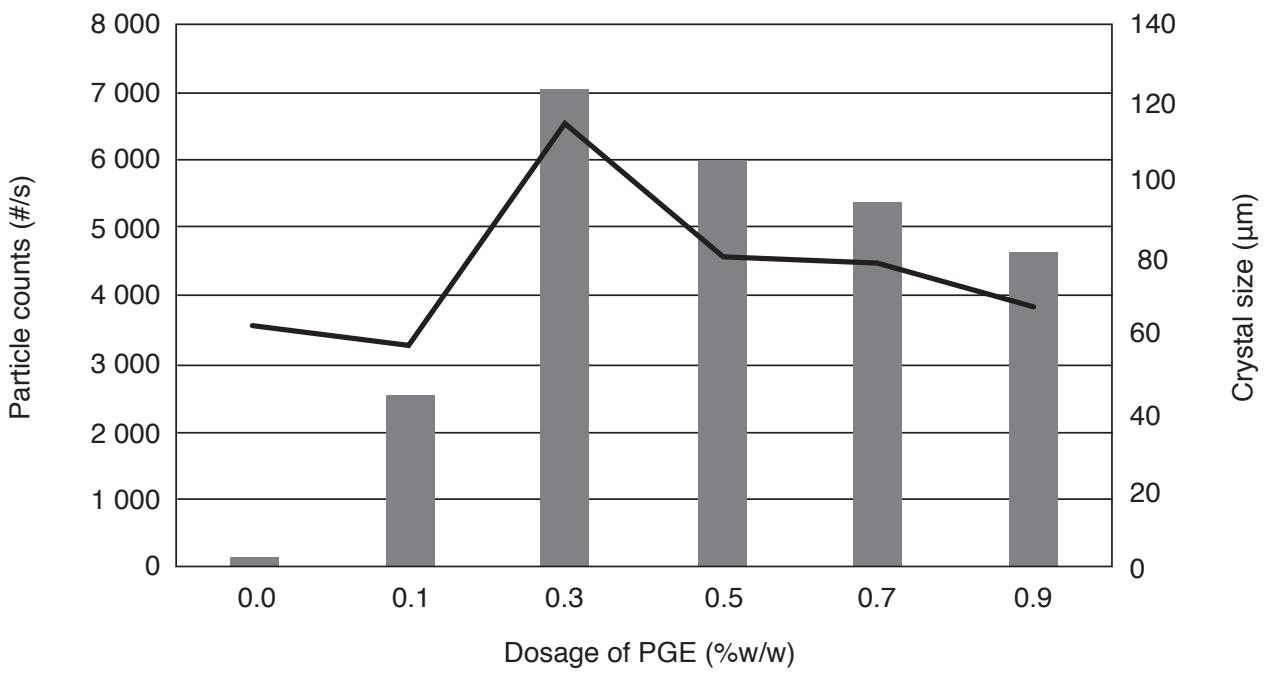

Dosage of PGE $(\% w / w)$

Particle counts $\quad$ Particle size

Note: PGE - polyglycerol ester.

Figure 1. Particle counts and mean particle size during the final step of isothermal crystallisation $\left(470 \mathrm{~min}, 13^{\circ} \mathrm{C}\right)$.

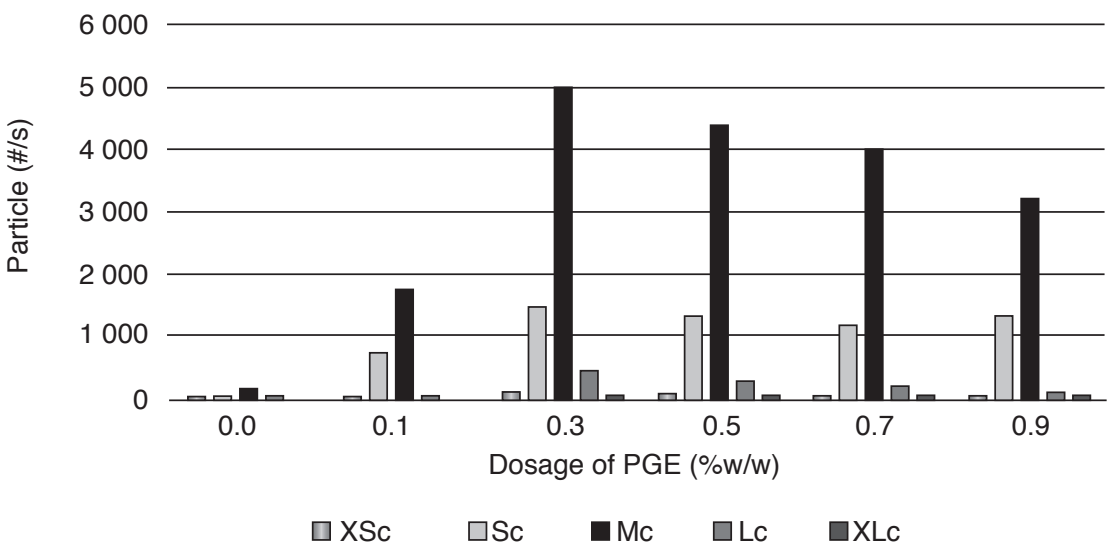

Note: PGE - polyglycerol ester.

Figure 2. Total particle counts in different crystal sizes during the final step of isothermal crystallisation (470 min, $\left.13^{\circ} \mathrm{C}\right) . \mathrm{XSc}-1-5 \mu \mathrm{m}$; Sc-10-23 $\mu \mathrm{m} ; \mathrm{Mc}-29-86 \mu \mathrm{m} ; \mathrm{Lc}-100-251 \mu \mathrm{m} ;$ and, XLc-293-1000 $\mu \mathrm{m}$. 
monitored. During this process, isothermal holding time was expressed as from 0-135 min. The crystal growth during the isothermal holding was monitored using FBRM. The FBRM measured various weighted distributions which emphasise different size ranges.

Figure 3 shows that PGE dosages enhance nucleation and increase crystal populations as compared with crystallisation without PGE. Particle counts for control was the lowest during the $135 \mathrm{~min}$ of isothermal holding time. Nucleation for control only occurred at $130^{\text {th }}$ min when the isothermal holding time was extended to $160 \mathrm{~min}$ as shown in Figure 4. This indicates that the PGE dosages reduced time for nucleation by $65 \mathrm{~min}$ from $130 \mathrm{~min}$ without PGE to $60 \mathrm{~min}$ with PGE. Dosage at $0.3 \%(\mathrm{w} / \mathrm{w})$ had the highest particle counts with a higher increasing rate. Steepest slope was obtained at $0.3 \%(\mathrm{w} / \mathrm{w})$ than the other dosages, indicating that $0.3 \%(\mathrm{w} / \mathrm{w})$ promotes nucleation. The particle counts and particle increasing rate of $0.5 \%(\mathrm{w} / \mathrm{w})$ and $0.7 \%(\mathrm{w} / \mathrm{w})$ were similar. Dosage at $0.9 \%(\mathrm{w} / \mathrm{w})$ has higher increasing particle rate in the beginning at $60 \mathrm{~min}$ but the rate reduced after $90 \mathrm{~min}$, indicating minor retardation of nucleation. PGE behaved as a foreign surface in the RBDPOo to promote the primary heterogeneous nucleation in which the increased particle counts and earlier nucleation were observed. Similar effect was not found in the control where no PGE was added. Primary and homogenous nucleation occurred in control RBDPOo, normal crystallisation of fat where the nucleation was not catalysed by fat crystals or foreign solid surfaces (Gunstone et al., 2007). This finding agrees with the study by Saw et al. (2017) where nucleation was enhanced in the crystallisation of palm oil when PGE dosages at

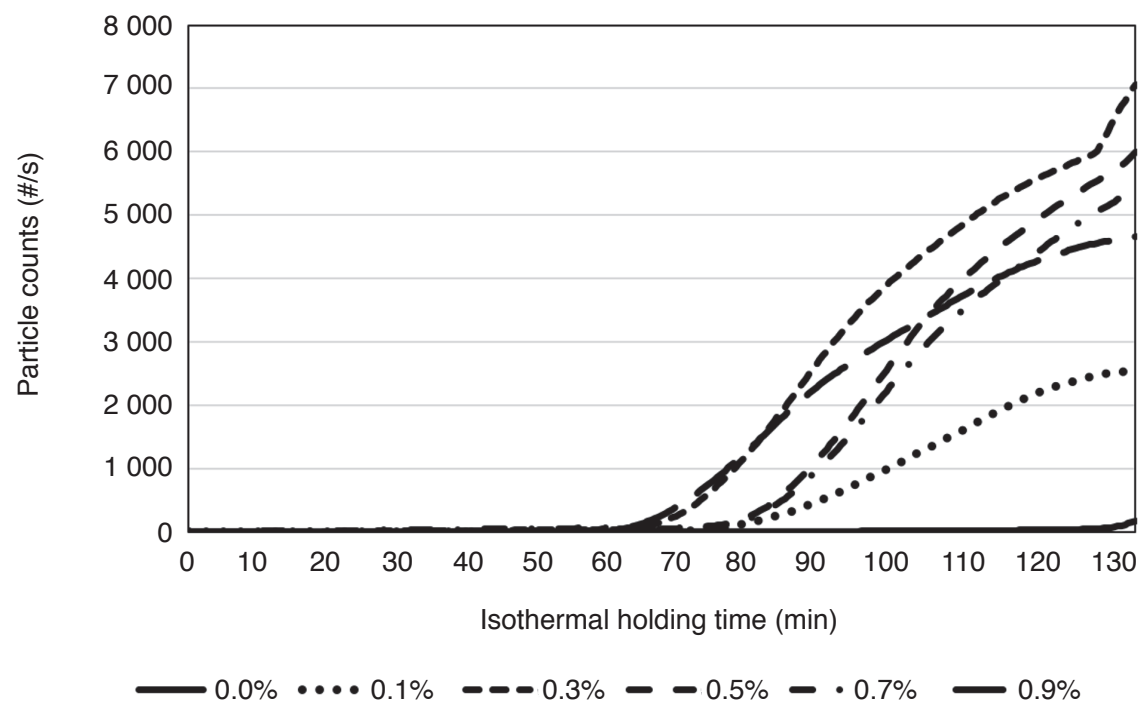

Figure 3. Changes of particle counts during isothermal crystallisation.

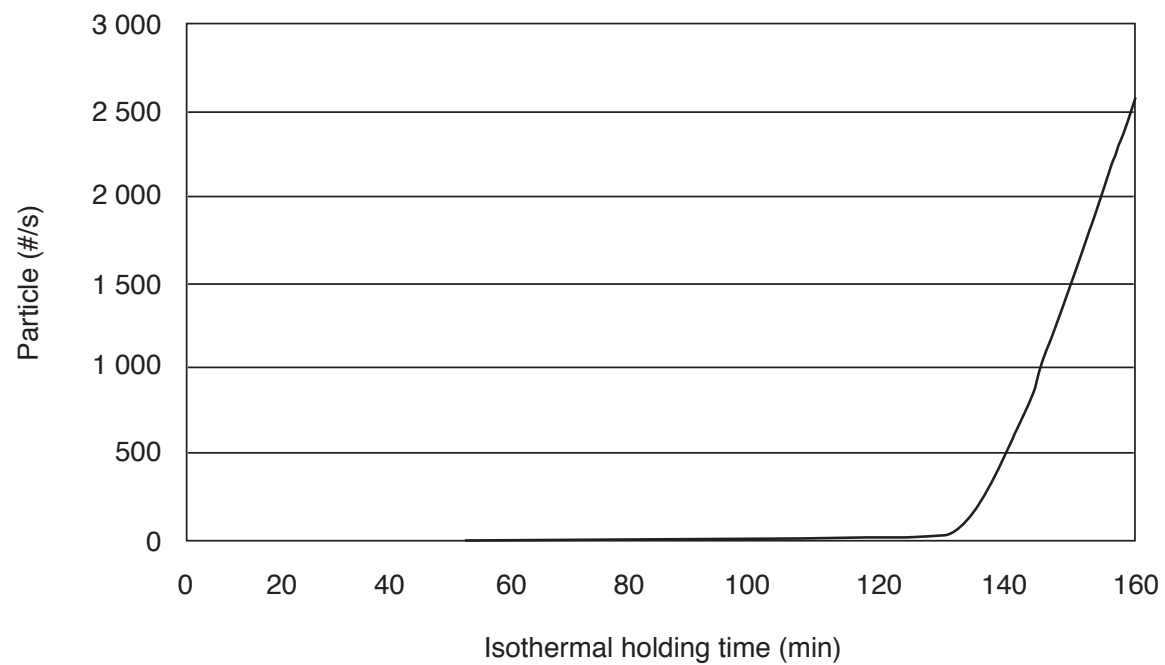

Figure 4. Changes of particle counts during extended isothermal holding time for $0 \%$ (w/w) of polyglycerol ester dosage. 
$0.1 \%(\mathrm{w} / \mathrm{w})-0.7 \%(\mathrm{w} / \mathrm{w})$ were added. The study by Saw et al. (2017) observed that the rate of increase of crystals increased with higher dosages of PGE.

Similar study done by Hachiya et al. (1989) using 1,3-behenoyl-2-sn-oleoyl-glycerol (BOB) as a seed to promote crystallisation of stearic-oleicstearic acids (SOS) in cocoa butter confirmed our present findings in RBDPOo crystallisation. The BOB seeds accelerated crystallisation of cocoa butter. Similar observation also can be seen in the present study where addition of PGE promotes early formation of crystals in RBDPOo (Figure 3). However, the fractionation enhancer used in the current study was not a crystal seeding process as it did not appear in solid form when it was added into RBDPOo. During crystal seeding, foreign crystal particles are added during the cooling period of crystallisation process when PGE is added and melted with RBDPOo before crystallisation process commences. PGE presented itself as a homogeneous solution in the RBDPOo providing catalytic seeding effects to form the primary and heterogeneous nucleation in crystallisation. The seeding effects of PGE is mainly attributed by the fatty acid structure (palmitic, stearic and oleic acids) of PGE which is similar to POP in RBDPOo, that helps to promote the crystal formation of POP (Saw et al., 2017).

\section{Effects of PGE on Crystal Growth}

Figure 5 shows changes of crystal size distribution and crystal growth during the isothermal holding time. Medium and smaller size crystals were mainly found in the $0 \%(\mathrm{w} / \mathrm{w})$ PGE dosage (Figure 5a). Medium size crystals in control formed at $90 \mathrm{~min}$, however, the amount of crystals was not significant. The medium size crystals formed at a slower rate than those with PGE dosages. Crystal growth occurred when nuclei were formed and exceeded the critical size during nucleation (Gunstone et al., 2007). The late nucleation in the RBDPOo crystallisation without the presence of PGE caused slower crystal growth. The lower rates of nucleation caused the formation of smaller amount of medium and smaller size crystals when compared to PGE additives.

Figures $5 b$ to $5 f$ show the evolution of particles in different size ranges of RBDPOo slurries during $135 \mathrm{~min}$ of isothermal crystallisation with dosage of $0.1 \%(\mathrm{w} / \mathrm{w}), 0.3 \%(\mathrm{w} / \mathrm{w}), 0.5 \%(\mathrm{w} / \mathrm{w}), 0.7 \%$ $(\mathrm{w} / \mathrm{w})$ and $0.9 \%(\mathrm{w} / \mathrm{w})$ PGE. The figures generally show PGE dosage accelerated the nucleation of RBDPOo as compared to the control. The RBDPOo crystallisation with or without PGE dosage formed similar crystal sizes, which were mainly smaller size crystals $(10-23 \mu \mathrm{m})$ and medium size crystals (29-86 $\mu \mathrm{m})$. The number of crystals increased proportionally to the isothermal holding time for all dosages of PGE. The $0.9 \%(\mathrm{w} / \mathrm{w})$ had a slower rate as compared with other PGE dosages. Dosage at 0.3\% $(\mathrm{w} / \mathrm{w})$ had 5000 count/s of crystals with 29-86 $\mu \mathrm{m}$ and 1500 count/s of 10-23 $\mu \mathrm{m}$ crystals, having the highest count/s of crystals among the others. Normah et al. (2013) suggested that crystals with size ranging from 4.34 to $29.29 \mu \mathrm{m}$ are stable and uniform and play a pivotal role in reducing olein entrainment through better filtration performance. Saw et al. (2017) also concurred that smaller crystals and more even in size offer better filtration performance where possibility of olein entrainment can be reduced. Dosage at $0.3 \%(\mathrm{w} / \mathrm{w})$ exhibited formation of more small and medium crystals and retarded formation of large and extra large crystals. The crystal size of $0.3 \%(\mathrm{w} / \mathrm{w})$ was more even as compared with the others. However, the sizes of increased crystal amounts observed in this present study were slightly different from the crystal sizes suggested by Normah et al. (2013) and Saw et al. (2017) due to different process where the previous studies were done in palm oil crystallisation which contained more saturated fatty acid with higher melting points (tripalmitoyl-glycerol, PPP). The present study on olein crystallisation contained mainly POP which lowers the melting points (Hishamuddin and Omar, 2016).

\section{IV of the Fractionation Products}

Figure 6 shows the IV of the products after the fractionation of RBDPOo viz. DFPOo and PMF. The IV of the DFPOo with $0.3 \%(\mathrm{w} / \mathrm{w})$ and $0.5 \%(\mathrm{w} / \mathrm{w})$ were the highest among the others at $58.62 \mathrm{~g} / 100 \mathrm{~g}$ and $58.82 \mathrm{~g} / 100 \mathrm{~g}$, respectively. The fractionation with $0.3 \%(\mathrm{w} / \mathrm{w})$ added PGE had the PMF with the lowest IV at $52.5 \mathrm{~g} / 100 \mathrm{~g}$. In general, the IV of DFPOo were slightly increased when PGE was added into the fractionation. In the case of PMF, IV were found to decrease when PGE was added where more POP was found in the solid fraction. It appears that adding PGE improved the crystallisation and filtration of RBDPOo, resulting in higher IV for DFPOo and lower IV for PMF. PGE additives helped enhance the crystallisation by inhibiting the growth of larger crystals which may contribute to the occurrence of olein entrainment. This was because the larger crystals tend to group together in clumps and occlude oleins (Hishamuddin and Omar, 2016). These findings also concur with the study on effects of PGE on palm oil fractionation done by Kuriyama et al. (2011). The previous study showed that PGE helped to reduce entrained olein in the stearin (lower IV in solid fractions) which resulted in higher IV in the DFPOo.

In general, the PGE with $0.3 \%(\mathrm{w} / \mathrm{w})$ promoted reduction in crystal sizes and increased population of smaller and medium size crystals provided better PMF and DFPOo as compared with the other dosages. 
(a)

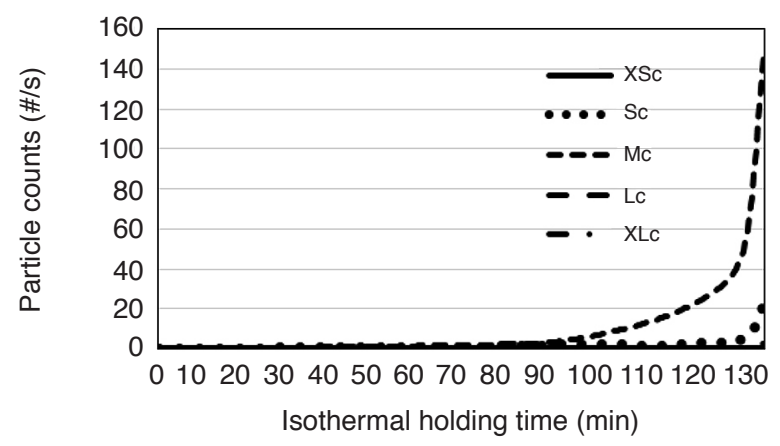

(b)

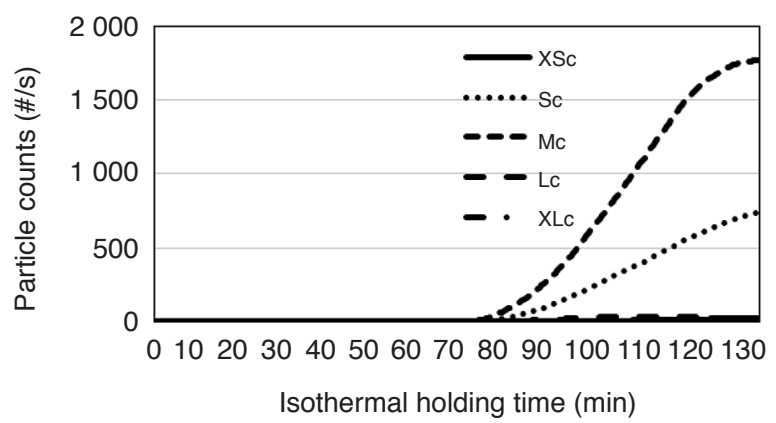

(c)

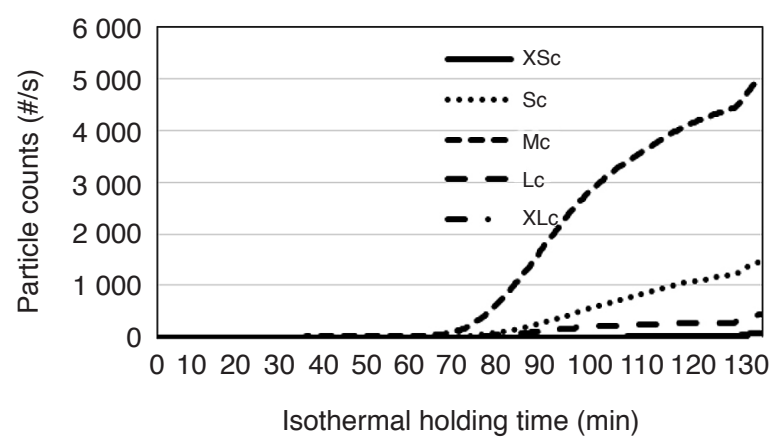

(d)



(e)



(f)

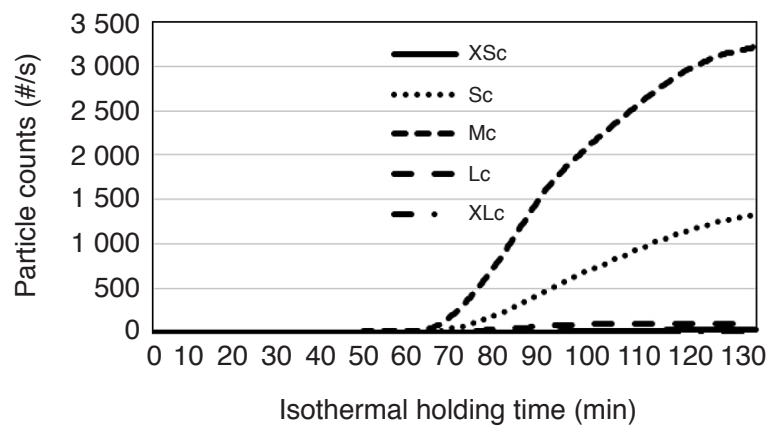

Figure 5. Evolution of particle counts in different size ranges of palm olein slurries during 135 min of isothermal crystallisation with (a) $0 \%$ (w/w), (b) $0.1 \%(w / w),(c) 0.3 \%(w / w),(d) 0.5 \%(w / w),(e) 0.7 \%(w / w)$ and $(f) 0.9 \%(w / w)$ polyglycerol ester dosages.

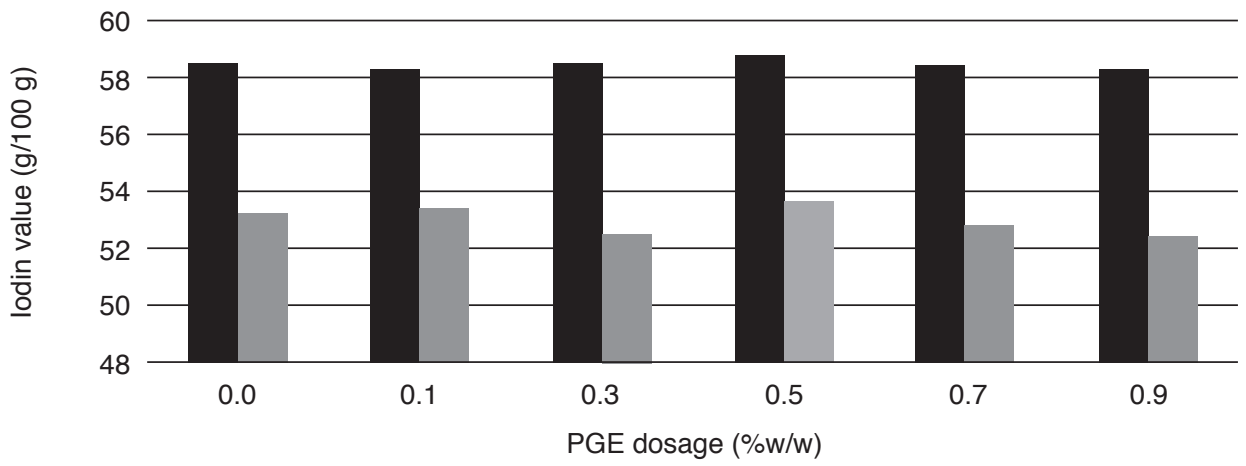

DF $\quad$ PMF

Figure 6. Iodine value of double fractionated palm olein (DFPOo) and palm mid fraction (PMF) with different polyglycerol ester (PGE) dosages. 


\section{TAG Analyses of the Fractionation Products}

To confirm the findings on olein entrainment in PMF, a TAG analysis was carried out for the products. Figure $7 a$ shows that there was no observable significant difference between the total amounts of $\mathrm{POO}+$ palmitoyl-linoleoyl-oleoylglycerol (PLO) and POP, and dosages of PGE in DFPOo. All PGE dosages gave similar percentage of POP and total percentage of POO/PLO in DFPOo (Figure 7a). It appears that PGE did not significantly affect the TAG content of DFPOo. DFPOo from the fractionation with or without PGE dosages have similar TAG composition. The observation of olein entrainment is normally being detected in PMF instead of DFPOo. This is because of the existence of olein in the PMF due to olein entrainment will cause changes to the TAG distribution of PMF. The entrainment, however, does not occur in the liquid fraction and therefore no significant difference can be observed in TAG distribution in the DFPOo.

In the TAG analysis on PMF, the POO and PLO of TAG represented the unsaturated TAG in the oil while the POP represented the saturated TAG. Due to similar melting point with POP, oleins with the formation of PLO and POO often entrap in the PMF (Sato, 2001). In the case of PMF, POO and POP contents improved when the amount of PGE was added. POO of PMF decreased when dosage of PGE increased. On the other hand, the POP of PMF decreased when PGE dosage increased.

In the case of PMF as shown in Figure $7 b$, there were differences in the POP and total percentage of PLO+POO between the PMF obtained from fractionations with different PGE dosages. The POP in PMF added with $0.3 \%(\mathrm{w} / \mathrm{w})$ was highest among the others while having the lowest content of $\mathrm{PLO}+\mathrm{POO}$. The lowest $\mathrm{PLO}+\mathrm{POO}$ content indicates the lowest oil entrainment in the PMF with $0.3 \%(\mathrm{w} / \mathrm{w})$ PGE dosage. PGE at $0.5 \%(\mathrm{w} / \mathrm{w})$ dosage and above showed negative impact to the TAG contents in the PMF (lower POP and higher content of $\mathrm{PLO}+\mathrm{POO})$. The findings agree with the study by Kuriyama et al. (2011) which concluded that dosing of certain amount of PGE helps enhance the fractionation process by reducing the incidence of olein entrainment in solid fractions. The findings also agree with the study by Saw et al. (2017) that showed a little amount of PGE helps enhance the crystallisation process.

\section{Crystallisation Mechanism}

The possible mechanism involved in this crystallisation process is that the PGE molecules act as the material for the cluster formation, and therefore accelerate nucleation when $0.3 \%(\mathrm{w} / \mathrm{w})$ of PGE was added. PGE's effect on RBDPOo crystallisation was mainly attributed by its composition of palmitic acid, stearic acid and oleic acid, like majority of RBDPOo fatty acid composition. These fatty acids have similar chain length, saturation, and type of unsaturation (Smith et al., 2001). This explains even a very low dosage of PGE, i.e. $0.1 \%(\mathrm{w} / \mathrm{w})$ was able to give relatively significant effect on crystal growth.

The nucleation occurs when the oil becomes supercool or reaches the point where the oil temperature is lower than the thermodynamic equilibrium temperature (Kellens et al., 2007). Crystals are generated when nucleation occurs. Meanwhile, the crystal growth occurs after nucleation when crystals formed in nucleation grows together to form a crystal lattice. However, in most cases, both nucleation and crystal growth (a)

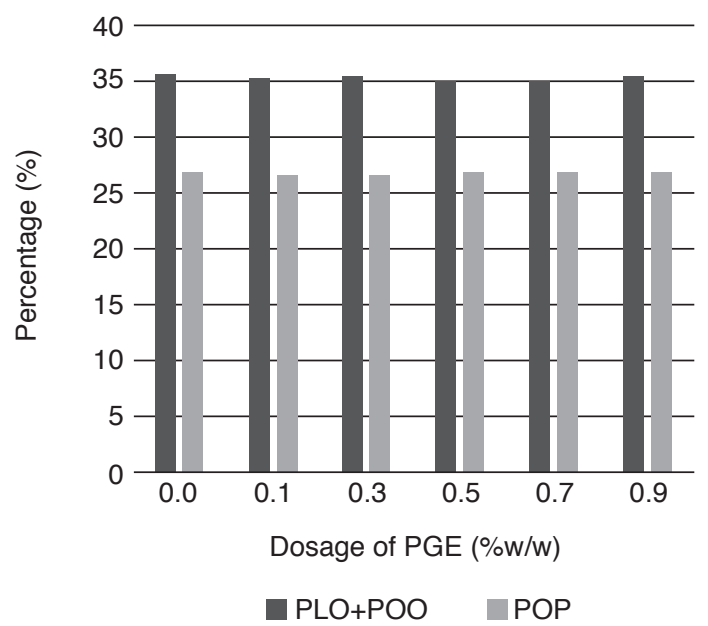

(b)

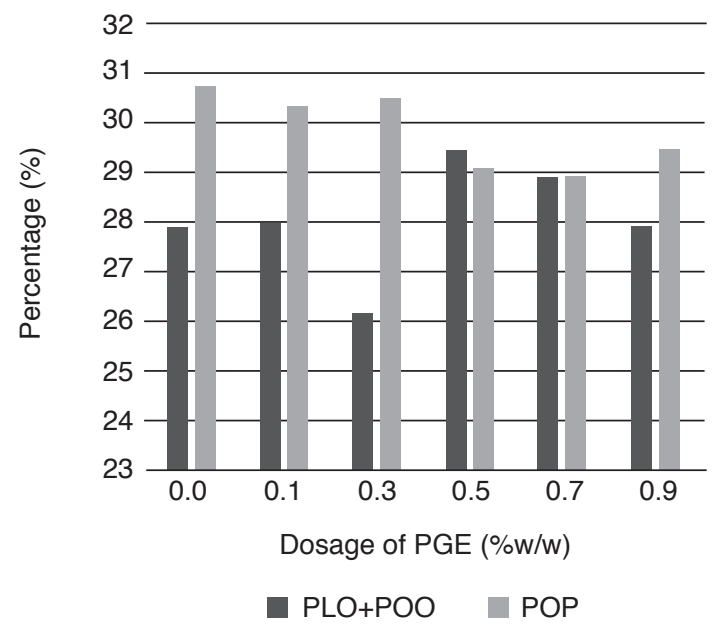

Figure 7. Palmmitoyl-linoleoyl-oleoyl-glycerol+1-palmitoyl-2, 3-oleoyl-glycerol (PLO+POO) and 1,3-dipalmitoyl-2oleyl-glycerol (POP) contents in (a) double fractionated palm olein (DFPOo) and (b) palm mid fraction (PMF) obtained from fractionations with different polyglycerol ester (PGE) dosages. 
occur side by side (Metin and Hartel, 2005). In crystallisation, the combination of nucleation and crystal growth leads to the formation of crystals with different size distributions. PGE accelerated the nucleation by forming a surface for initiating the primary, heterogeneous nucleation. The enhanced nucleation further promotes the increased formation of crystallites from nuclei (crystal growth). The promotional effects by PGE is mainly attributed by its similar structure to RBDPOo and its higher melting point. However, previous study showed that PGE inhibits the growth of larger crystals which helps in avoiding the occurrence of olein entrainment. The saturated fatty acids in PGE promotes the formation of medium crystals while unsaturated fatty acids increase the numbers of larger crystals (Zhang et al., 2016). In this regard, it can be deduced that PGE promotes nucleation and crystal growth to form small and medium crystals but it inhibited growth of larger crystals. The crystal growth rates for $0 \%$, $0.1 \%, 0.3 \%, 0.5 \%, 0.7 \%$ and $0.9 \%$ are $0.07 \mu \mathrm{m} \cdot \mathrm{min}^{-1}$, $-0.10 \mu \mathrm{m} \cdot \mathrm{min}^{-1}, 0.61 \mu \mathrm{m} \cdot \mathrm{min}^{-1}, 0.27 \mu \mathrm{m} \cdot \mathrm{min}^{-1}, 0.29$ $\mu \mathrm{m} \cdot \mathrm{min}^{-1}$ and $0.05 \mu \mathrm{m} \cdot \mathrm{min}^{-1}$, respectively.

The current study agrees with the findings reported by Hachiya et al. (1989) in crystallisation of cocoa butter using BOB. The study suggested that the seed materials with saturated fatty acids and higher melting points effectively promote crystal nucleation. In the case of current study using PGE with melting point of $42.8^{\circ} \mathrm{C}$, positive effects on crystal nucleation and formation were observed. PGE molecules act as foreign surface that enhances the formation of nuclei in the RBDPOo crystallisation (Sato et al., 1999). Sato (2001) suggested that stearic acid prevents formation of eutectic phase for the mixture of POP-POO. The eutectic phase for the mixture of POP-POO is often a challenge for filtration or separation of PMF (POP-dominant solid phase) from DFPOo (POOdominant liquid phase) in fractionation process. PGE with appreciable amounts of stearic acid showed the above effect and this can be observed when the POP content in the PMF was higher for fractionation with PGE addition as compared to the fractionation of control.

Based on Smith et al. (2011), the effects of additive with respect to nucleation are changes of nucleation time, shift in nucleation temperature and changes in the number and nature of nuclei formed. However, due to the device limitations, many techniques for nucleation measurements do not detect nucleation directly as the nuclei sizes are too fine and beyond the detectable range. The nuclei have to grow into crystals to be detectable (Smith et al., 2011). However, by using FBRM which is able to detect fine particles the size of even $1 \mu \mathrm{m}$, the early detection of crystal formation can be deduced to predict the nucleation. Therefore, information such as total particle count at the beginning of crystallisation can be related to nucleation (Saw et al., 2017).

The present study agrees with the study conducted by Kuriyama et al. (2011) which found that RBDPOo with PGE additive forms smaller crystals as compared with control. Smaller and uniform crystal size reduces aggregation to form granular crystals, thereby reducing the entrainment of olein in the stearin (Kuriyama et al., 2011). An increased amount of smaller crystals in $0.3 \%(\mathrm{w} / \mathrm{w})$ explains the higher POP content and lower POO content in PMF produced from $0.3 \%(\mathrm{w} / \mathrm{w})$.

The observations on RBDPOo with higher dosages of PGE, i.e. $0.3 \%(\mathrm{w} / \mathrm{w}), 0.5 \%(\mathrm{w} / \mathrm{w}), 0.7 \%$ $(\mathrm{w} / \mathrm{w})$ and $0.9 \%(\mathrm{w} / \mathrm{w})$ provided better nucleation and better control in crystal growth as compared with the $0 \%(\mathrm{w} / \mathrm{w})$ and $0.1 \%(\mathrm{w} / \mathrm{w})$. This observation agrees with the study done by Saw et al. (2017). The previous study showed that in palm oil fractionation, when more PGE additive was used, significant enhancement of heterogeneous nucleation was observed (Saw et al., 2017). In this present study in RBDPOo fractionation, heterogeneous nucleation occurred in the control and 0.1\% (w/w) PGE without significant interferences from the additive molecules. The physico-chemical analysis of the fractionation products shows that $0.3 \%(\mathrm{w} / \mathrm{w})$ PGE fractionation produced better PMF where the products have lower IV and lesser olein entrainment (less PLO+POO).

\section{CONCLUSION}

The study concludes that PGE additive provided multiple effects on the enhancement of crystallisation in RBDPOo fractionation, particularly on the nucleation, crystal growth of RBDPOo and physicochemical properties of the fractionation products. The effects of PGE additive varied with different dosage levels and $0.3 \%(\mathrm{w} / \mathrm{w})$ was found to be the optimum dosage for RBDPOo fractionation. PGE additive accelerated the crystal nucleation, and this can be observed in all dosages of PGE. The accelerated nucleation is useful in the fractionation process as it helps lower cost of production by reducing the hour of fractionation by $65 \mathrm{~min}$ as compared with $130 \mathrm{~min}$ without PGE additive. However, PGE retarded the growth of undesired larger crystals and promoted the formation of smaller crystals and more even crystal sizes. This enhances the fractionation process by limiting entrainment of olein in PMF. Olein entrainment is often the factor that caused inefficient fractionation where POO, which are supposed to be in DFPOo phase, were trapped in PMF. The reduction of olein entrainment and enhanced POP content in PMF help to reduce production cost and increase product values. 


\section{ACKNOWLEDGEMENT}

The authors thank the Director-General of MPOB for permission to publish this article.

\section{REFERENCES}

Deffense, E (1985). Fractionation of palm oil. J. Amer. Oil Chem. Soc., 62: 376-385.

Ghazali, H M; Hamidah, S and Che Man, Y B (1995). Enzymatic transesterification of palm olein with nonspecific and 1,3-specific lipases. J. Amer. Oil Chem. Soc., 72: 633-639.

Gunstone, F D; Harwood, J L and Dijkstra, A J (2007). The Lipid Handbook. Third edition. CRC Press.

Hachiya, I; Koyano, T and Sato, K (1989). Seeding effects on solidification behaviour of cocoa butter and dark chocolate. I. Kinetics of solidification. J. Amer. Oil Chem. Soc., 66: 1757-1762.

Hishamuddin, E and Omar, Z (2016). In situ characterisation of palm olein crystallisation behaviour by focused beam reflectance measurement (FBRM). J. Oil Palm Res. Vol. 28: 44-51.

Kellens, M; Gibon, V; Hendrix, M and Greyt, W D (2007). Palm oil fractionation. European J. Lipid Science and Technology, 109: 336-349.

Kuriyama, J; Miyamoto, Y and Sakamoto, M (2001). Effect of polyglycerol fatty acid esters on resistance to crystallization of palm olein. J. Oleo Science, 50: 831-838.

Kuriyama, J; Miyaji, Y; Tamura, K; Zaliha, O and Chong, C L (2011). Improved sustainable fractionation of palm oil using polyglycerol fatty acid esters. J. Oil Palm Res.Vol. 23: 1141-1145.

Metin, S and Hartel, R W (2005). Crystallization of fats and oils. Bailey's Industrial Oil and Fat Products. Sixth edition. p. 6.

MPOB (2005). MPOB Test Methods. MPOB, Bangi. p. 3.

Normah, I; Cheow, C S and Chong, C L (2013). Crystal habit during crystallization of palm oil: Effect of time and temperature. International Food Research J., 20(1): 417-422.
Sakamoto, M; Ohba, A; Kuriyama, J; Maruo, K; Ueno, S and Sato, K (2004). Influences of fatty acid moiety and esterification of polyglycerol fatty acid esters on the crystallization of palm mid fraction in oil-in-water emulsion. Colloids and Surfaces B: Biointerfaces, 37: 27-33.

Sakamoto, M; Fukunaga, K; Kuriyama, J and Sato, K (2005). Retardation effects of polyglycerol fatty acid esters on the crystallization of n-hexadecane. J. Oleo Science, 54(4): 211-216.

Sato, K (2001). Crystallization behaviour of fats and lipids. Chemical Engineering Science, 56: 2255-2265.

Sato, K; Ueno, S and Yano, J (1999). Molecular interactions and kinetic properties of fats. Progress in Lipid Research, 38: 91-116.

Saw, M H; Hishamuddin, E; Chong, C L; Yeoh, C B and Lim, W H (2017). Effect of polyglycerol esters additive on palm oil crystallization using focused beam reflectance measurement and differential scanning calorimetry. Food Chemistry, 214: 277-284.

Smith, K W (2001). Crystallization Processes in Fats and Lipid Systems (Garti, N and Sato, K eds.). Mercel Dekker, Inc. p. 357-380.

Smith, K W; Bhaggan, K; Talbot, G and Van Malssen, K F (2011). Crystallization of fats: Influence of minor components and additives. J. Amer. Oil Chem. Soc., 88: 1085-1101.

Swe, P Z; Che Man, Y B and Ghazali, H M (1995). Composition of crystal of palm olein formed at room temperature. J. Amer. Oil Chem. Soc., 72: 343-347.

Suria, A Y (1996). Refining and downstreaming processing of palm and palm kernel oils. Selected Readings on Palm Oil and its Uses (Abdullah A ed.). PORIM, Bangi. p. 35-49.

Timms, R E (2005). Fractional crystallization - The fat modification process for the $21^{\text {st }}$ century. European J. Lipid Science and Technology, 107: 48-57.

Yu, Z Q; Chow, P S and Tan, R B H (2008). Interpretation of focused beam reflectance measurement (FBRM) data via stimulated crystallization. Organic Process Research \& Development, 12: 646-654.

Zhang, N; Yang, X; Teng, Y L; Wan, F L; Fu, J N and Wang, Y (2016). Modern Food Science and Technology, 32(3): 48-55. 